\title{
Integración de imágenes del sensor MODIS y cartografía temática para la simulación de modelos geoespaciales para obtener zonas propensas a desertificación en el Estado de Sinaloa, México
}

\author{
Miguel Armando López Beltrán* \\ Juan Martín Aguilar Villegas** \\ Wenseslao Plata Rocha***
}

Recibido el 18 de diciembre de 2015; aceptado el 6 de agosto de 2016

\begin{abstract}
Desertification is a complex process, involving environmental and anthropogenic factors in a dry climate context. This process has a global importance, because it reduces the productivity and value of causing an impact on the territory and its population. The information technology helps identify areas prone to desertification through geospatial and quantitative indicators. This research therefore seeks to develop a methodology that allows to model the input variables obtained from MODIS sensor data, thematic mapping and statistical information, which is then integrated from multicriteria evaluation technique weighted linear sum. The variables were considered are: increased albedo, reduce biomass, deforestation, spearly vegetated areas; vegetated areas (green cover); moisture content in the soil; precipitation; surface temperature; distance of human assessment, agriculture, hidrology networks; areas with physical and chemical degradation and areas with water and wind erosion. The weighting is performed with the method of Saaty pairwise comparison. To provide robustness to generated model, it proceeded to perform a validation of the result (desertification index) using land use and vegetation maps, climate and humidity, arid index and intensity of soil degradation. Desertification

* Tesista, Universidad Autónoma de Sinaloa, México, correo electrónico: miguel.armandolb@gmail.com

** Director de tesis, Universidad Autónoma de Sinaloa, México, correo electrónico: a_villegas@uas.edu.mx

*** Director de tesis, Universidad Autónoma de Sinaloa, México, correo electrónico: wenses@uas.edu.mx
\end{abstract}


index show that most vulnerable areas are in the northern of Sinaloa and reduced southward.

Key words: Desertification, MODIS, Thematic Cartographic, Multicriteria Evaluation, GIS.

\section{Resumo}

A desertificação é um processo complexo, que envolve fatores ambientais e antropogênicos dentro de um contexto climático seco. Dispõe de uma importância global, que reduz a produtividade e o valor dos solos ocasionando um impacto sobre o território e sua população. As Tecnologias de Informação ajudam a identificar zonas propensas a desertificação através de indicadores geoespaciais e quantitativos. Nesta pesquisa se definiu como objetivo o desenvolvimento de uma proposta metodológica que permite modelar as variáveis de entrada obtidas a partir de dados do sensor MODIS, cartografia temática e informação estatística, que posteriormente se integram a partir de avaliação multicritério com a técnica de somátoria linear ponderada. Se consideraram as seguintes variáveis de entrada: aumento de albedo, diminuição de biomassa, desmatamento, zonas com escassa vegetação e com vegetação, contendo umidade no solo, precipitação, temperatura superficial, distâncias a assentamentos humanos, zonas agrícolas, redes hidrológicas, zonas com degradação e erosão de solos. A ponderação se realizou com o método de comparação por pares de Saaty. Para dar robustez ao modelo gerado, se realizou uma validação dos resultados utilizando mapas de uso de solo e vegetação, climas e umidade, índice de aridez e intensidade de degradação de solos. Os resultados obtidos mostram que as áreas mais vulneráveis a desertificação são a zona norte do Estado de Sinaloa e se reduz de acordo com o avanço para o sul.

Palavras chave: Desertificação, MODIS, Cartografia temática, Avaliação multicritério, SIG.

\section{Resumen}

La desertificación es un proceso complejo, involucra factores ambientales y antropogénicos dentro de un contexto climático seco. Dispone de una importancia global, dado que reduce la productividad y el valor de los suelos ocasionando un impacto sobre el territorio y su población. Las Tecnologías de Información ayudan a identificar zonas propensas a desertificación a través de indicadores geoespaciales y cuantitativos. En esta investigación se planteó como objetivo el desarrollo de una propuesta metodológica que permita modelar las variables de entrada obtenidas a partir de datos del sensor MODIS, cartografía temática e información estadística, que posteriormente se integran a partir de evaluación multicriterio con la técnica sumatoria lineal ponderada. Se consideraron las siguientes variables de entrada: aumento de albedo, disminución de biomasa, deforestación, zonas con escasa vege- 
tación y con vegetación, contenido de humedad en el suelo, precipitación, temperatura superficial, distancias a asentamientos humanos, zonas agrícolas, redes hidrológicas, zonas con degradación y erosión de suelos. La ponderación se realizó con el método de comparación por pares de Saaty. Para dar robustez al modelo generado, se procedió a realizar una validación de los resultados utilizando mapas de uso de suelo y vegetación, climas y humedad, índice de aridez e intensidad de degradación de suelos. Los resultados obtenidos muestran que las zonas más vulnerables a desertificación son en la zona norte del Estado de Sinaloa y se reduce acorde del avance hacia el sur.

Palabras clave: desertificación, MODIS, Cartografía temática, evaluación multicriterio, SIG.

\section{Introducción}

La desertificación es un proceso de degradación gradual donde interactúan distintos entes clasificados en factores antropogénicos (agricultura, ganadería, actividades forestales, desarrollo humano, cambios de uso de suelo, etc.) y variaciones ambientales (variación de albedo, altas temperaturas, baja humedad, suelos sin vegetación, aridez, sequías, etc.) dentro de un contexto climático de regiones áridas a subhúmedas secas siendo un proceso irreversible (Santini y otros 2010).

Al principio de la década del 2000, este fenómeno global afectaba aproximadamente un $40 \%$ de la superficie terrestre. Asimismo, una superficie de 36 millones de $\mathrm{km}^{2}$, equivalente a un $70 \%$ de las tierras áridas, estaba afectada en algún grado de desertificación, afectando a más de 1,000 millones de personas, ocasionando pérdidas de $\$ 42$ mil millones de dólares anuales (Holtz, 2003; SEMARNAT, 2008; $\mathrm{Xu}, \mathrm{y}$ otros 2010). Actualmente, este proceso está afectando entre 5-10 millones de hectáreas anualmente solo en el sector agrícola (Bakr y otros 2012). Según un reporte de la Convención de las Naciones Unidas de la Lucha contra la Desertificación (UNCCD, 2014) menciona que aproximadamente 135 millones de personas se encuentran en riesgo de ser desplazados por la desertificación por lo que el combate contra este problema tiene un costo mundial de $\$ 490$ mil millones de dólares (UNCCD, 2013).

\section{Desertificación en México}

En México, se han realizado evaluaciones sobre este fenómeno. En 1991, la Comisión Nacional de Ecología (CNE), presentó información cuantitativa y cualitativa sobre la desertificación realizada en 1981, elaborado por la Secretaría de Desarrollo Urbano y Ecología (SEDUE), tomando como base criterios topográficos, erosión, precipitación y aptitud del suelo para la agricultura y ganadería, dando como resultado que casi todo el territorio presentaba cierto grado de vulnerabilidad (CNE 1991). En 1995, el Insituto Nacional de Ecología (INE) estimó el 96.98\% del 
territorio nacional como vulnerable a la desertificación en distintos grados (Oropeza 2004). La Secretaria de Medio Ambiente y Recursos Naturales (SEMARNAT) en 1999, estimó que aproximadamente el $64.03 \%$ se encontraba en un estado de degradación de suelo, relacionando la degradación con desertificación. En el año 2001, Semarnat y el Colegio de Posgraduados estimaron que el $45.20 \%$ del territorio se encontraba en un grado de desertificación, relacionando la degradación en el contexto climático de regiones áridas (Oropeza 2004; SEMARNAT, 2008).

Para el año 2005, México conformó un Sistema de Lucha Contra la Desertificación y la Degradación de los Recursos Naturales (SINADES), integrado por distintas dependencias del sector público, social y académico. (SEMARNAT, 2013). Sin embargo, según SEMARNAT para el año 2008, no existían estudios específicos sobre la extensión de la desertificación a nivel nacional, pero se tenía una idea de la magnitud de ésta considerando la degradación del suelo en el contexto climático (SEMARNAT, 2008).

Se han realizado diversos trabajos de investigación sobre la desertificación en México. Hernández y otros (2009), realizaron un estudio del territorio nacional sobre desertificación, a partir de cartas de climas obtenidas del portal de CONABIO (Comisión Nacional para el Conocimiento y Uso de Biodiversidad), a escala 1:1,000,000 del año 1998. Los resultados presentados mencionan que el 31.5\% del territorio nacional presenta algún grado a desertificación.

Ivanova y Gámez (2012), presentan una estimación a desertificación en Baja California Sur por lo que se utilizaron como indicadores: la aridez, pendiente, orientaciones, texturas de suelo, coberturas vegetales, degradación y erosión de suelos, condiciones de los acuíferos, pastoreo, caminos, zonas agrícolas, zonas urbanas y áreas naturales protegidas; y para la integración de los factores un modelo multicriterio en donde cada identificador fue estandarizado, priorizado y ponderado, conforme a la comparación por pares de Saaty y siguiendo el método del análisis jerárquico (AHP).

Otro estudio, realizado en Cuatro Ciénegas, Coahuila por Madrigal y López (2009), utilizan imágenes del sensor Landsat ETM+ para obtener los indicadores de Índice de Vegetación de Diferencia Normalizada (NDVI), Índice de Desviación Estándar Dinámico (MSDI) y albedo, integrando todas las variables al método de clasificación de árbol de decisión. También, se realizó una evaluación de los procesos de desertificación para una cuenca en el noreste de México considerando solamente como indicador la erosión hídrica por Pando y otros (2002). Por último, se tiene el trabajo realizado por Norzagaray y otros (2011), en el municipio de Guasave, Sinaloa, utilizando como indicadores las variables de: $\mathrm{pH}$ (potencial de Hidrogeniones), densidad aparente y real, sólidos totales disueltos, conductividad eléctrica y los niveles de concentración de aluminio, cloruro, fierro, magnesio y manganeso; y como técnica las series de Fourier para obtener sus respectivos espectro de potencia y radial. 


\section{Metodologías e indicadores utilizados para estudios de desertificación}

Existe una gran diversidad de investigaciones enfocadas en el estudio de desertificación. Todos ellos aplicando diversos métodos, fuentes y variables en percepción remota y Sistemas de Información Geográfica. Navone y otros (2002), utilizaron ocho indicadores obtenidos de observaciones de campo, fotografías aéreas, imágenes satelitales de Radar-Sat 1 y Landsat, determinando la desertificación a partir de una sobreposición entre los datos. Liu y otros (2003), utilizaron el coeficiente de variación del NVDI obtenido del AVHRR (Advance Very High Resolution Radiometer). Qin y otros (2004), estimaron la degradación de la cubierta vegetal a partir de observaciones de campo, cambios de uso de suelo agrícola e imágenes MODIS (NVDI), evaluando una relación directa entre la degradación de la cubierta y la desertificación. Changzhen y otros (2005), a partir del movimiento de arena y los valores de NVDI estimaron con un árbol de decisión la desertificación, utilizaron imágenes del sensor MODIS y Landsat. Guo y Li (2005), monitorearon un tipo de desertificación denominada como desertificación arenosa (sandy desertification) utilizaron imágenes Landsat (años 1986 y 2000), mapa de desertificación arenosa y mapa de usos de suelos. Li y otros (2007) determinaron zonas con desertificación a partir de imágenes Landsat, utilizando como indicador los usos de suelo. Fang y otros ( 2008), utilizaron dos imágenes del sensor Landsat (1986 y 2000), con una clasificación supervisada para determinar. Zhang y otros (2008), desarrollaron una metodología que permita el seguimiento de desertificación en Yulin, China. Para ello, utilizaron como insumo mapas topográficos e imágenes Landsat generando dos bases de datos. La primera base de datos fue de geografía básica. La segunda es una base de datos sobre el suelo desertificado. Meléndez y otros (2009), determinaron el potencial de utilizar la percepción remota para los estudios del proceso de desertificación. Yan y otros (2009), estimaron con un árbol de decisión la desertificación a partir de los insumos de erosión eólica y porcentaje de la cubierta vegetal. Xu y otros (2009), utilizaron como indicadores el potencial de la producción primaria neta (NPP por sus siglas en inglés) y la diferencia entre potencial y el actual NPP. Li y otros (2009), utilizaron el análisis de correlación Grey (Grey Correlation Analisys). Hao y Zhao (2010), analizaron los efectos de los tipos de usos de suelos y su conversión a desertificación. Kasimu y Tateishi (2010), usaron el método de clasificación por árbol de decisión. Santini y otros (2010), generaron un índice de desertificación integrando modelos de pastoreo, productividad vegetativa, fertilidad del suelo, intrusión del agua de mar, erosiones hídricas y eólicas. Huo y otros (2011), proponen un método para obtener mediciones de campo, que permite identificar zonas con desertificación de manera precisa a partir de datos de albedo superficial. Duanyang y otros (2012), utilizaron el árbol de decisión para estimar desertificación a partir de cubierta vegetal, albedo y contenido de humedad en el suelo. Ferrara y otros (2012), utilizaron 14 
indicadores que fueron aplicados en el índice de áreas ambientalmente sensibles. Petta, y otros (2013), utilizaron datos de precipitación e imágenes de NDVI de MODIS, datos Landsat ETM+, datos de campo, mapas temáticos (pendiente, tipos de suelos, morfología, etc.) para obtener un mapa de zonas degradadas y susceptibles a desertificación.

Asimismo, existen trabajos elaborados bajo un modelo ya establecido denominado MEDALUS (Meditarranean Desertification and Land Use), como es el caso de Farajzadeh y Egbal (2007), Rasmy y otros (2010), Gad y Shalaby (2010), Bakr y otros(2012), estableciendo ciertas modificaciones al modelo.

\section{Área de estudio}

El Estado de Sinaloa (Figura 1) se encuentra ubicado en la parte noroeste de México, en las coordenadas geográficas extremas de: $27^{\circ} 02^{\prime}$ y $22^{\circ} 29^{\prime}$ latitud Norte y $105^{\circ} 23^{\prime}$ y $109^{\circ} 25^{\prime}$ longitud Oeste. Colinda en la costa con el Golfo de California y el océano Pacífico al Oeste, el Estado de Sonora al Norte, Chihuahua y Durango al Este, y al Sur con Nayarit teniendo un rango de altitudes de 0 a 2,520 msnm. Los climas van desde templados subhúmedo a muy seco muy cálido y cálido. La temperatura media anual es de $24.86^{\circ} \mathrm{C}$, con precipitaciones medias acumuladas de $674.44 \mathrm{~mm}$ anuales. Dispone de 19 corrientes y 7 cuerpos de agua. Los principales tipos de vegetación son: pastizales, bosques, selvas, matorrales, agricultura, entre otros menos predominantes (INEGI, 2012).

\section{Propuesta metodológica}

\section{Datos y metodología}

Con el fin de identificar zonas vulnerables a desertificación en el Estado de Sinaloa, México, se seleccionaron algunos indicadores como principales agentes catalizadores en el proceso (Tabla 1). Se seleccionó dos años de estudio (2003 y 2012).

Se elaboró un mapa de restricción mediante el mapa de división política de Sinaloa, las zonas marítimas, redes hidrológicas, carreteras y asentamientos humanos para ambos años. Las variables fueron transformadas a la proyección UTM$13 \mathrm{~N}$, a una resolución espacial aproximada de $250 \mathrm{~m}$.

Se utilizó como técnica de evaluación multicriterio la sumatoria lineal ponderada, que determina los niveles de vulnerabilidad al sumar el resultado de multiplicar la ponderación del factor por la ponderación del criterio:

$$
r_{i}=\sum_{j=1}^{n} w_{\underline{j}} v_{i j}
$$

Donde: $r_{i}$ es el nivel de adecuación del factor $i ; w_{j}$ es el peso del criterio $(j) ; v_{i j}$ es el valor ponderado del factor $i$ en el criterio $j$. 


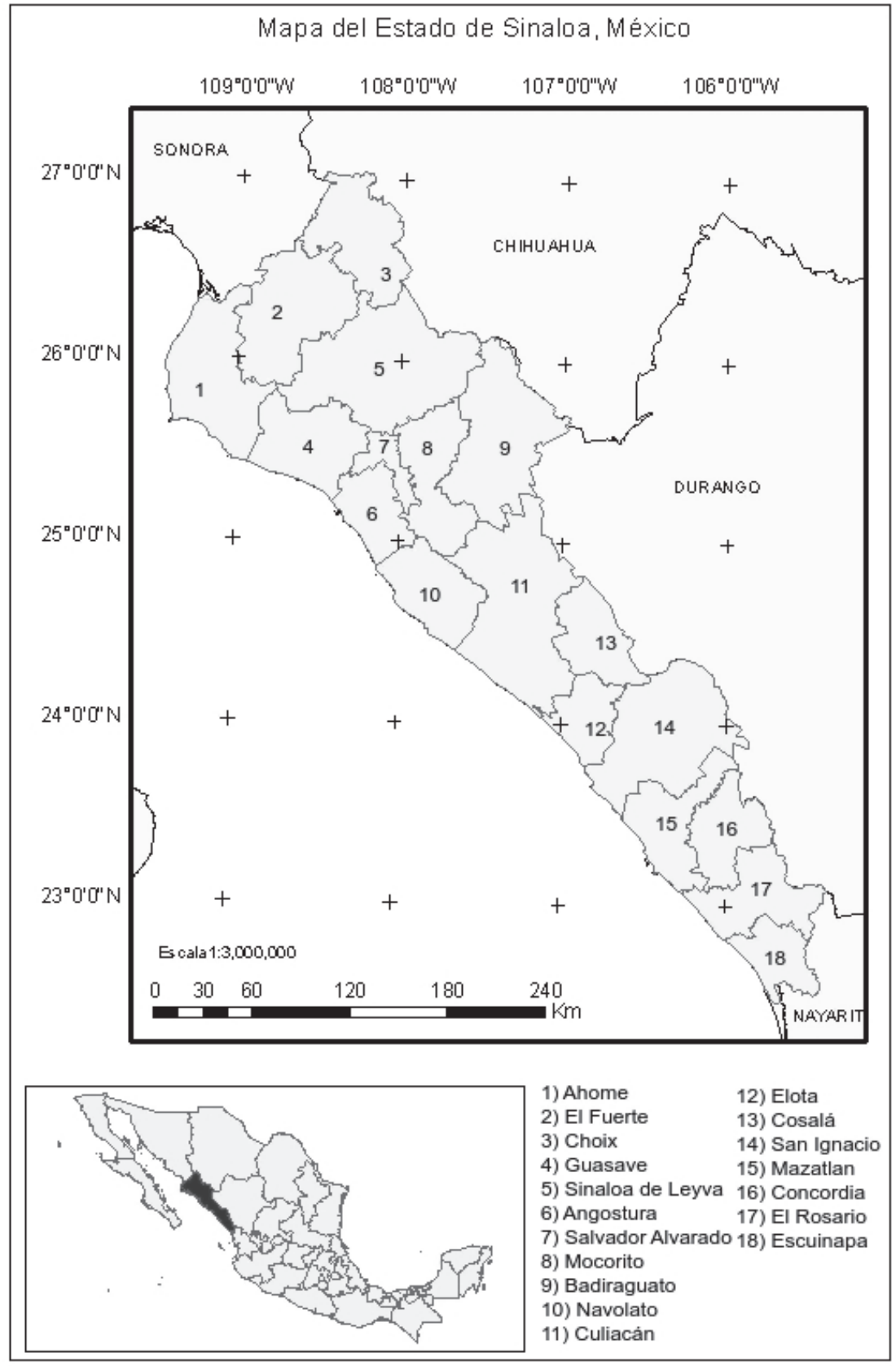

Figura 1. Mapa del área de estudio representativo del Estado de Sinaloa. 
Tabla 1

Jerarquía entre criterios y factores

\begin{tabular}{llll}
\hline Criterio & Descripción & Factores & Fuente \\
\hline \multirow{4}{*}{ Ambiental } & $\begin{array}{l}\text { Factores bióticos y } \\
\text { abióticos que pueden } \\
\text { influir en el equilibrio } \\
\text { de los ecosistemas en } \\
\text { forma directa e } \\
\text { indirecta }\end{array}$ & $\begin{array}{l}\text { Aumento de albedo } \\
\text { Disminución de } \\
\text { biomasa } \\
\text { Temperatura superfi- } \\
\text { cial }\end{array}$ & $\begin{array}{l}\text { MOD } \\
\text { MOD }\end{array}$ \\
\hline Antropogénicos & $\begin{array}{l}\text { Factores relacionados } \\
\text { con toda actividad } \\
\text { humana. }\end{array}$ & $\begin{array}{l}\text { Cercanía a } \\
\text { asentamientos } \\
\text { humanos } \\
\text { Cercanía a carreteras }\end{array}$ & CT \\
Cercanía a zonas \\
Humedad
\end{tabular}

MOD: Productos derivados del sensor MODIS.

CT: Cartografía temática.

EM: $\quad$ Estaciones meteorológicas.

Fuente: Elaboración propia.

\section{Generación de variables}

\section{MODIS}

Como insumo en percepción remota, se utilizaron los productos del sensor MODIS, siguiendo un algoritmo de procesamiento: 1) Descarga de datos para los años de 
estudio (2003 y 2012); 2) Importación y conversión de los archivos HDF a ráster del software; 3) Delimitación a la zona de estudio; 4) Elaboración de una variable anual a partir de algebras de mapas; 5) Aplicación de filtro de cuadro adaptativo para cambiar valores fuera de rango o sin información; 6) Asignación de la proyección UTM-13N y homogenización a una resolución espacial aproximado de 250m; 7) Homogenización entre las diferentes unidades de medida de los factores (normalización) con el módulo Fuzzy en el rango de escala de bits (0-255). En la realización de los procesos anteriores se utilizó el software IDRISI Selva.

\section{Cartografía temática}

Se descargó cartografía temática desde el portal de INEGI (Instituto Nacional de Estadística y Geográfica) para representar ciertos factores (asentamientos humanos, carreteras, redes hidrológicas, degradación y erosión de suelos).

El algoritmo de procesado para obtener los factores se realizó con los softwares ArcGIS 9.3 e IDRISI Selva. En ArcGIS se realizaron los procesos para delimitar la zona de estudio y georreferencias los mapas a la zona 13 Norte de la proyección UTM. Posteriormente, se importó el archivo vectorial al software IDRISI para su rasterización y asignación de los mismos parámetros de las imágenes MODIS.

\section{Datos compuestos}

Existen variables que juegan un papel importante en la desertificación; sin embargo, algunas requieren ser generadas a partir de distintos insumos, o en algunos casos enriquecerse de distintas fuentes. El contenido de humedad del suelo se calculó con el índice de sequedad-temperatura vegetación (TVDI - Temperature-Vegetation Dryness Index). Para asentamiento humano se compilo la información de diferentes fuentes para generar una imagen compuesta. Por último, la precipitación fue generada a partir de una interpolación entre los datos de pluviómetros de estaciones meteorológicas.

\section{Validación de los resultados}

Para dar robustez a los resultados obtenidos se consideraron cuatro aspectos diferentes: uso de suelo y vegetación, climas y humedad, índice de aridez e intensidad de degradación. La validación estará basada en dos criterios: estadística descriptiva para datos nominales y regresión lineal para datos cuantitativos.

\section{Resultados}

Se obtuvo una base de datos que consiste en 16 variables, todas ellas con diferentes resoluciones espaciales y formato; 16 factores y un mapa de restricción en formato 
ráster en una resolución espacial de aproximadamente de 250 metros cuya matriz es de 1,622 columnas y 2,045 filas.

\section{Ponderación}

En la ponderación de factores, se establecieron primero los pesos para los criterios, posteriormente se ponderó a cada factor dentro de cada criterio. Finalmente se obtuvo el peso final al multiplicar el peso del criterio con el peso de cada factor (Tabla 2).

Tabla 2

Valores de ponderación para criterios y factores

\begin{tabular}{|c|c|c|c|c|}
\hline Criterios & Peso & Factores & Peso & Peso final \\
\hline \multirow[t]{3}{*}{ Ambiental } & 0.0667 & Aumento de albedo & 0.5407 & 0.0361 \\
\hline & & Disminución de biomasa & 0.2966 & 0.0198 \\
\hline & & Temperatura superficial & 0.1627 & 0.0109 \\
\hline \multirow[t]{3}{*}{ Antropogénico } & 0.2666 & Cercanía a asentamientos humanos & 0.2857 & 0.0762 \\
\hline & & Cercanía a carreteras & 0.1429 & 0.0381 \\
\hline & & Cercanía a zonas agrícolas & 0.5714 & 0.1523 \\
\hline \multirow[t]{3}{*}{ Humedad } & 0.2003 & Lejanía a redes hidrológicas & 0.5407 & 0.1083 \\
\hline & & Precipitación & 0.2966 & 0.0594 \\
\hline & & Contenido de humedad en el suelo & 0.1627 & 0.0326 \\
\hline \multirow[t]{3}{*}{ Vegetación } & 0.1330 & Deforestación & 0.2966 & 0.0394 \\
\hline & & Índice de vegetación & 0.5407 & 0.0719 \\
\hline & & Suelos sin o con escasa vegetación & 0.1627 & 0.0216 \\
\hline \multirow[t]{4}{*}{ Suelo } & 0.3334 & Degradación física & 0.3333 & 0.1111 \\
\hline & & Degradación química & 0.3333 & 0.1111 \\
\hline & & Erosión eólica & 0.1667 & 0.0556 \\
\hline & & Erosión hídrica & 0.1667 & 0.0556 \\
\hline
\end{tabular}

\section{Evaluación multicriterio: índice de zonas propensas a desertificación (IZPD)}

Esta técnica evalúa a cada factor dentro de los criterios establecidos, a partir de una valoración cuantitativa. En el resultado de EMC, los valores más elevados representan las zonas con mayor vulnerabilidad a presentar desertificación. El mapa resultante se le denominó como índice de zonas propensas a desertificación (IZPD). El IZPD en el año 2003 mostró como resultado un valor mínimo de 59 y un máximo de 209, con una media de 151.63. Para el caso del año 2012, los valores mínimos y máximos son de 59 y 212, respectivamente, con una media de 152.44. 
Para ambos años (Figura 2) muestra un comportamiento similar, teniendo una correlación de 0.97. Para dar veracidad a este índice y determinar la vulnerabilidad en el Estado respecto al fenómeno se realizó una prueba de robustez (validación) para determinar si es viable o no este índice.
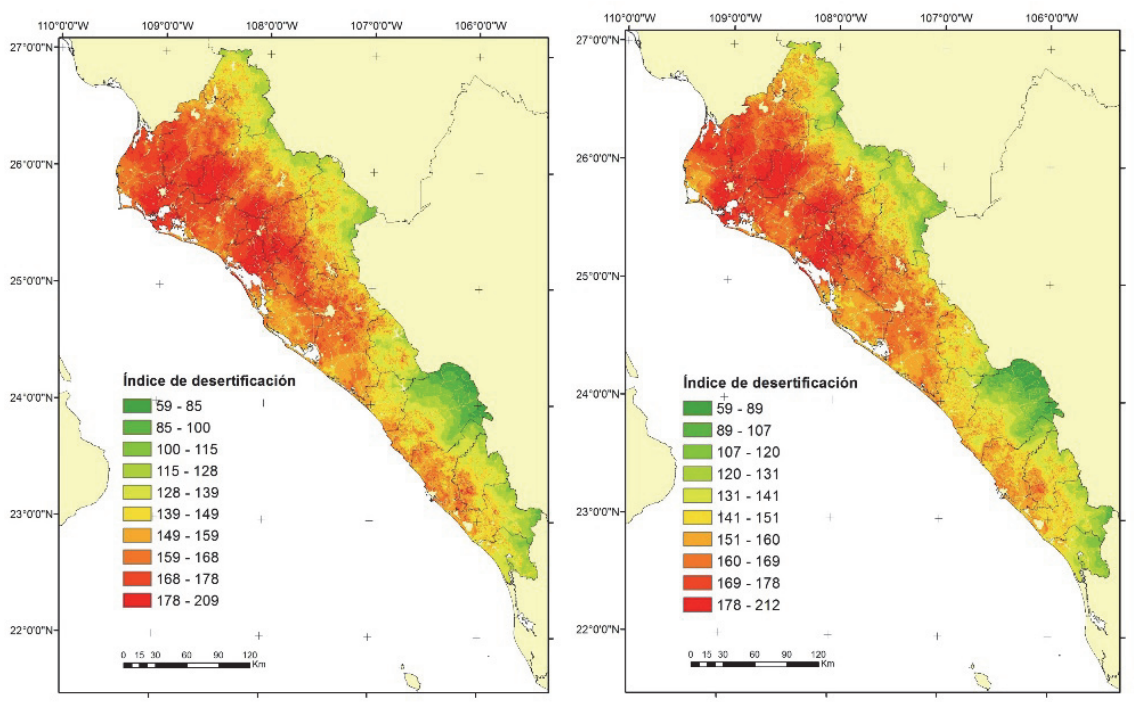

Figura 2. Mapas de vulnerabilidad a desertificación. Derecha año 2003 e izquierda año 2012.

\section{Validación}

Validación con mapas de uso de suelo y vegetación e índice de vegetación

La validación se realizó extrayendo los valores medios del IZPD con respecto a las cubiertas de uso de suelo. Los resultados obtenidos muestran que las clases más vulnerables son los matorrales, pastos y cultivos. Estos se encuentran en espacios más abiertos y en los climas más secos, además la densidad de vegetación es menor dejando desprotegido el suelo en algunas áreas, lo que permite sea el más vulnerable a desertificación por condiciones climáticas y fisiográficas. La categoría de cultivos representa la cubierta antropogénica que sufre mayor impacto de acuerdo con las referencias bibliográficas. Asimismo, el IZPD va disminuyendo de acuerdo con las diferentes cubiertas, que disponen y cumplen con ciertas características ambientales que disminuye el IZPD. El valor medio más alto es en matorrales abiertos para ambos años (171.20 y 171.27) seguidos de cultivos (166.81 y 166.20) y pastos (164.66 y 163.66). 

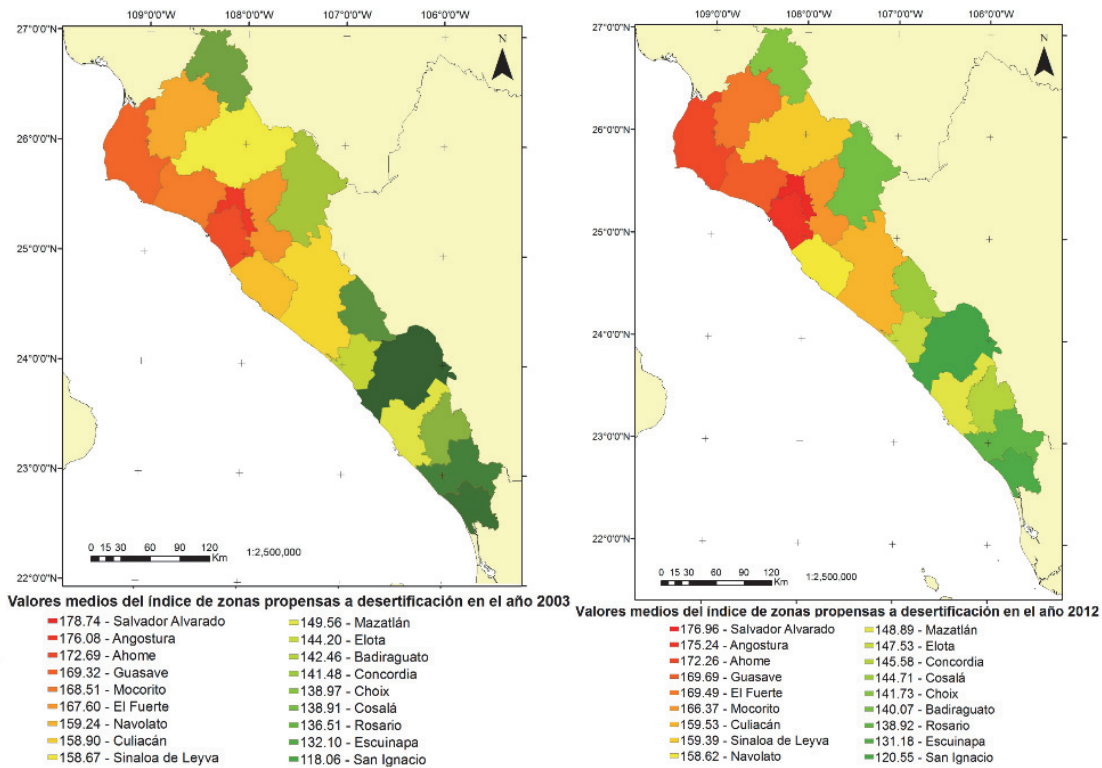

Figura 3. Mapa de valores medios del índice de zonas propensas a desertificación a nivel municipal. Izquierda año 2003 y derecha año 2012.
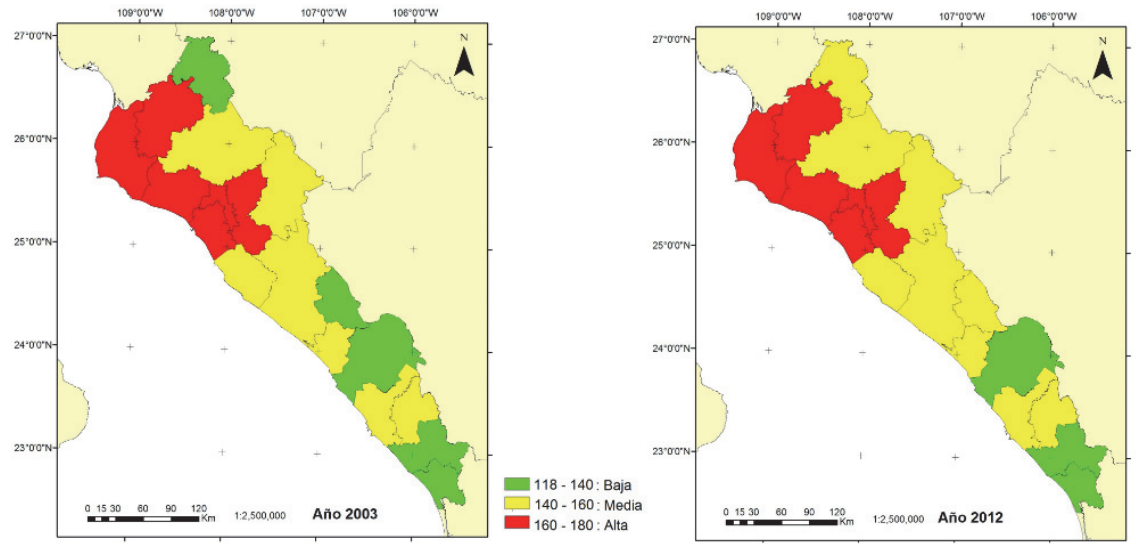

Figura 4. Discretización a nivel municipal de los valores del índice de zonas propensas a desertificación en tres niveles ordinales. Izquierda año 2003 y derecha año 2012. 
Se utilizó el índice de vegetación (NDVI) para correlacionarlo mediante una regresión lineal y el IZPD, obteniendo coeficientes de -0.63 y -0.56 para los años 2003 y 2012, respectivamente. Lo anterior muestra que el IZPD disminuye mientras aumenta el valor de NDVI, determinando que a menor densidad de vegetación existe mayor probabilidad de presentar suelos con desertificación.

\section{Validación con mapas de climas}

El identificador del mapa de climas está en forma ordinal, donde el valor más bajo son climas más áridos. Se realizó una regresión lineal y los coeficientes son de -0.68 y -0.69 para 2003 y 2012 respectivamente. Como se estimó, los valores medios más altos se encuentran en los climas áridos.

\section{Validación con mapas de aridez}

Con el mapa de aridez (fórmula de DeMartonne), se realizó una regresión lineal con el IZPD bajo la hipótesis de valores menores del índice, mayores valores del IZPD. Los coeficientes arrojados por la regresión lineal son -0.70 y 0.28 para el año 2003 y 2012. Par el año 2003 muestra una relación alta, sin embargo, no es el caso para el año 2012, posiblemente la baja relación se deba a la variación de la precipitación en ese año o la cantidad de estaciones utilizadas para la interpolación en una resolución de $250 \mathrm{~m}$.

\section{Validación con mapas de intensidad de degradación}

El grado de intensidad, presenta también un comportamiento similar de aumento en el nivel de vulnerabilidad con los valores medios del IZPD. Los valores medios del IZPD mostraron que a medida que aumenta el nivel de vulnerabilidad aumenta la intensidad de degradación en los suelos.

\section{Zonas propensas a desertificación a nivel municipal}

Los resultados obtenidos a nivel municipal se muestran en las Figura 3. Los valores medios altos se muestran para ambos años (2003 y 2012) en los municipios en la zona norte ubicados en la costa y valle del Estado, debido, principalmente, a las condiciones antropogénicas. Mientras que los valores medios más bajos en ambos años se dan en los municipios que se localizan gran parte de su territorio enclavados en la zona serrana, aunado a las condiciones climáticas y redes hidrográficas. Los municipios de la zona centro-norte y centro-sur del estado se encuentran con valores intermedios posiblemente por las condiciones climáticas moderadas de la región y la densidad de vegetación de la zona, asimismo, se observa una zona de transición entre la parte desértica del norte y la parte tropical del sur del Estado (Figura 4). 


\section{Conclusiones}

La creación de una base de datos geoespacial para el fenómeno de desertificación requiere tener actualizadas las variables que se están usando para dicho estudio. Asimismo, integrar variables en el enfoque socioeconómico.

La utilización de productos del sensor MODIS y su integración con otras variables temáticas de instituciones oficiales y/o privadas, así como de insumos generados, permiten tener datos actualizados, de calidad y a un costo económico y de tiempo muy bajo.

La modelación de las variables geoespaciales mediante análisis espacial requiere conocer la naturaleza de los datos y su representación anual.

Los resultados obtenidos muestran distribución esperada de acuerdo a lo que se conoce de la región. Con el uso de distintas épocas de estudio se puede determinar aquellas áreas o focos de atención, donde el proceso de desertificación puede ir avanzando y descartar aquellas zonas de coincidencia. Al conocer esto se pueden tomar medidas preventivas o de recuperación de los suelos.

Esta metodología puede ser considerada como la base para generar, integrar y modelar las variables geoespaciales, en diferentes escenarios de desertificación en un horizonte temporal futuro.

Basados en la validación de resultados permite sugerir que la metodología propuesta es un método viable para estimar la vulnerabilidad a desertificación.

\section{Trabajo futuro}

Realizar trabajos individuales para cada variable de entrada para determinar el rango de acción y calibración de valores que catalizan el desarrollo del fenómeno.

Explorar otras técnicas de análisis espacial y desarrollar metodologías de análisis de incertidumbre de los datos y análisis de sensibilidad de los modelos, con la finalidad de dar robustez a los resultados obtenidos en el modelo. Asimismo, desarrollar diferentes escenarios de desertificación a futuro para integrarse en modelos de cambio climático.

\section{Bibliografía}

Bakr, N.; Weindorf, D.C.; Bahnassy, M.H. and El-Badawi, M.M. (2012). "Multitemporal assessment of land sensitivity to desertification in a fragile agroecosystem: Environmental indicators", Ecological Indicators, vol. 15, no. 1, pp. 271-280.

Changzhen, Y.; Wang, T.; and Han, Z. (2005). "Using MODIS data to assess land desertification in ordos plateau - mu us sandy land case study", Geoscience and remote sensing symposium, no. 4, pp. 2373-2375. 
Chen, C.; Chen, C.; Son, N. and Chang, L. (2011). "Monitoring of soil moisture variability in relation to rice cropping systems in the vietnamese mekong delta using MODIS data”, Applied Geography, vol. 31, núm. 2, pp. 463-475.

Chuvieco Salinero, E. (2008). Teledetección ambiental: la observación de la Tierra desde el Espacio (3a. ed.), Ariel Ciencia.

CNE (1991). Informe de la situación general en la materia de equilibrio ecológico y protección al ambiente 1989-1990, Comisión Nacional de Ecología.

Farajzadeh, M. and Egbal, M. (2007). "Evaluation of MEDALUS model for desertification hazard zonation using GIS", Pakistan Journal of Biology Science, vol. 10, núm. 16, pp. 2622-2630.

Ferrara, A.; Salvati, L.; Sateriano, A. and Nole, A. (2012). "Performance evaluation and cost assessment of a key indicator systems to monitor desertification vulnerability", Ecological Indicators, núm. 23, pp. 123-129.

Gad, A. and Shalaby, A. (2010). "Assessment and mapping of desertification sensitive using remote sensing and GIS case study: Inland Sinai and Eastern desert Wadies, Cairo, Egipto", Space Technology and Geoinformation fo sustainable develoment.

Guo, M., and Li, X. (2005). "Monitoring sandy desertification of minqin oasis, northwest china by remote sensing and GIS", Geoscience and Remote Sensing Symposium no. 1, pp. 1-4.

Guzmán Fernández, D. (2010). Proceso de desertificación en el valle central de Tarija aplicando un modelo basado en indicadores espectrales, Universidad Mayor de San Simón, Vicerrectorado.

Hao, C. and Zhao, T.Q. (2010). "Study on relationship between landuse conversions and land desertificacition in mu us sandland region", 2nd conference on enviromental science and information application technology, pp. 440-442.

Hernández Cerda, M., Valdés Madero, G. y Miranda Salgado, B. (2009). "Proceso de desertificación", en J.R. Hernández Santana y B. Bollo Manent, I-II caracterización y diagnóstico para el ordenamiento eocológico general del territorio, SEMARNAT, INE, UNAM, UAQ, pp. 70-89.

Holtz, U. (2003). La convención de las Naciones Unidas de la lucha contra la desertificación (CNULD) y su dimensión política. Reporte de CNULD.

Holtzman, M.E. (2012). Estimación del estrés hídrico en cultivos y su relación con rendimientos en la región Pampeana mediante imágenes de temperatura de superficie e índice de vegetación, tesis doctoral.

Huo, A.; Xie, J.; Sun, Z. and Zhou, L. (2011). "Methodology of land surface broadband albedo retrieval in the desertification area based on MODIS image data", International symposium on water resource and enviromental protection, núm. 4, pp. 2539-2542. 
INEGI (2012). Anuario estadístico de Sinaloa 2012, Instituto Nacional de Estadística y Geografía.

Ivanova, A. y Gámez, A.E. (2012). Plan estatal de acción ante el cambio climático para Baja California Sur.

Kasimu, A. and Tateishi, R. (2010). "Extraction area at risk of desertification using MODIS and geophysical data: in xinjiang uyghur autonomous region of China", International conference on multimedia technology (ICMT), pp. 1-4.

Li, C.; Chen, X.; Chen, Y.; Fu, K. and Liu, Q. (2007). "Land desertification and some proposals for promoting sustainable development in Qinghai lake area, China", Geoscience and Remote Sensing Symposium, pp. 3444-34447.

Li, Z.; Jie, T.; Chenye, W. and Pingan, S. (2009). "Land desertification evaluation on the basis of quantitative remote sensing in Songnen plain China", 3rd international conference on bioinformatics and biomedical engineering, pp. 1-5.

Liu, A.; Liu, Z.; Wang, C.; Niu, Z. and Yan, D. (2003). "Monitoring of desertification in central Asia and western China using long term NOAAAVHRR NDVI time-series data", Geoscience and Remote Sensing Symposium 2003, no. 4, pp. 2278-2280.

Madrigal Gómez, J.M. and López Caloca, A. (2009). "Modelo de desertificación utilizando imágenes multiespectrales, en Cuatro Cienegas, Coahulia, México”. Ponencias en el XIV Simposio Internacional SELPER 2010.

Meléndez Pastor, I.; Navarro Pedraño, J.; Gómez, I. y Koch, M. (2009). “Análisis de series temporales de vegetación obtenidas mediante teledetección como herramienta para el seguimiento e procesos de desertificación", Congreso Internacional sobre desertificación en memoria del profesor John B. Thornes, pp. 339-342.

Navone , S., Espoz Alsina, C., Maggi, A. y Introcaso, R. (2002). "Monitoreo de la desertificación en los valles semiáridos del noroeste argentino: desarrollo de un sistema de información geográfica empleando indicadores biofísicos y socioeconómicos", Revista de Teledetección, núm. 18, pp. 5-19.

Norzagaray Campos, M.; Espinoza Carreón, L.; Muñoz Sevilla, P., y Rosales, A. G. (2011). "Condiciones físico-químicas del suelo y su asociación con la desertificación a través del análisis espectral en suelos del Valle de Gusave, Sinaloa", en N. Piedad Sánchez, G. Rosales Sosa, E.R. Santoyo Gutiérrez y M. Guevara García, XXI Congreso Nacional de Geoquímica, vol. 17, pp. 97-102.

Oropeza Orozco, O. (2004). "Evaluación de la vulnerabilidad a la desertificación", en J. Martínez, and A. Fernández Bremauntz, Cambio climático: una visión de México INE y SEMARNAT, pp. 301-312.

Pando Moreno, M.; Gutiérrez, G.M., Maldonado, H.A., y Jurado, E. (2002). "Evaluación de los procesos de desertificación en una cuenca hidrológica en el NE de México”, Ciencia UANL, vol. 5, núm. 4, pp. 519-524. 
Petta, R.; Vespoli de Carvalho, L.; Erasmi, S. and Jones, C. (2013). "Evaluation of desertification proccesses in Serido Region (NE Brazil)", International Jorunal of Geosciences, pp. 12-17.

Qin, Z.; Xu, B.; Xin, X.; Zhou, Q.; Zhang, H. and Liu, J. (2004). "Integration of remote sensing and GIS tecnology to evaluate grassland ecosystem health in Nort China", Geoscience and Remote Sensing Symposium, no. 6, pp. 4034-4037.

Rasmy, M.; Gad, A.; Abdelsalam, H. and Siwailam, M. (2010). "A dinamic simulation model of desertification of Egypt", The Egyptian Journal of remote sensing and space science, vol. 13, no. 2, pp. 101-111.

Ruíz Corral, J.; Medina García, G.; Macías Cervantes, J.; Silva Serna, M. y Díaz Padilla, G. (2005). Estadísticas climatológicas básicas del Estado de Sinaloa, Instituto Nacional de Investigaciones Forestales, Agrícolas y Pecuarias -INIFAP.

Santini, M.; Caccamo, G.; Laurenti, A.; Noce, S. and Valentini, R. (2010). "A multi-component GIS framework for desertification risk assessment by an integrated index", Applied Geographu, vol. 30, no. 3, pp. 394-415.

SEMARNAT (2008). Informe de la situación del medio ambiente en México: Compendio de estadísticas ambientales, México, Secretaría del Medio Ambiente y Recursos Naturales.

(2013). Informe de la situación del medio ambiente en México: compendio de estadísticas ambientales, indicadores clave y desempeño ambiental, (edición 2012), Secretaría de Medio Ambiente y Recursos Naturales.

UNCCD (2013). Land scan- what's new - 14-17/2013. Reporte en línea, United Nations Convention to Combat Desertification.

(abril de 2014). Desertificación: Esa invisible línea de frente (2da. edición), United Nations Convention to Combat Desertification, recuperado en abril de 2013.

Xu, D.; Kang, X.; Liu, Z.; Zhuang, D. and Pan, J. (2009). “Assessing the relative role of climate change and human activities in sandy desertification of ordos region China", Science in China Series D: Earth Sciencies, vol. 52, no. 6, pp. 855-868.

Yan, C.Z.; Song, X.; Zhou, Y.M.; Duan, H.C. and Li, S. (2009). "Assessment of aeolian desertification trens from 1975's to 2005's in the watershed of the longyangxia reservoir in the upper reaches of China's yellow river, Geomorphology, no. 112, pp. 205-211.

Zhang, C.; Chen, Z.; Zhu, B.; Luo, X.; Guan, Y.; Guo, S. and Nie, Y. (2008). "Land desertification monitoring and assessment in yulin of northwest China using remote sesing and geographic information systems (GIS)", Enviromental monitoring and assessment, vol. 147, nos. 1-3, pp. 327-337. 